\title{
SELETIVIDADE FISIOLÓGICA DE INSETICIDAS UTILIZADOS EM CULTURA CAFEEIRA SOBRE OVOS E ADULTOS DE CRYPTOLAEMUS MONTROUZIERI MULSANT
}

\section{L.C.D. Rocha ${ }^{1}$, G.A. Carvalho ${ }^{2}$, A.P. Moura ${ }^{3}$, V.F. Moscardini' ${ }^{2 *}$, D.T. Rezende ${ }^{4 * *}$, O.M. Santos $^{5 * *}$}

${ }^{1}$ Instituto Federal de Educação, Ciência e Tecnologia do Sul de Minas Gerais,Praça Tiradentes, 416, CEP 37576000, Inconfidentes, MG, Brasil. E-mail: luiz.rocha@ifs.ifsuldeminas.edu.br

\section{RESUMO}

Em cafeeiros, a associação de inimigos naturais com produtos fitossanitários seletivos é uma importante estratégia no manejo de pragas. Oobjetivo do presente estudo foi avaliar os efeitos letais e subletais de produtos fitossanitários utilizados na cultura cafeeira sobre ovos e adultos de CryptolaemusmontrouzieriMulsant em laboratório. Os bioensaios foram realizados sob $25 \pm 2{ }^{\circ} \mathrm{C}, \mathrm{UR}$ de $70 \pm 10 \%$ e fotofase de $12 \mathrm{~h}$. Os inseticidas utilizados e suas respectivas dosagens de aplicação, em g ou $\mathrm{mL}$ de i.a. $\mathrm{L}^{-1}$ foram: tiametoxam $(0,5)$, imidacloprido $(0,7)$, óleo mineral $(13,3)$, endossulfam $(2,63)$ e dimetoato $(0,48)$. A testemunha foi composta apenas por água destilada. A aplicação dos produtos foi realizada por meio de torre de Potter. Avaliaram-se a viabilidade de ovos, a sobrevivência dos espécimes e os efeitos dos compostos sobre os parâmetros reprodutivos do predador. Tiametoxam provocou prolongamento na duração do período embrionário (7,1 dias) em ovos tratados ejunto com imidacloprido foram classificados como nocivos, seguidos do endossulfam e dimetoato que apresentaram toxicidade intermediária e do óleo mineral que foi seletivo. Em função da seletividade do óleo mineral, pode-se recomendá-lo visando à compatibilização com o predador C. montrouzieri em programas de manejo integrado de pragas na cultura cafeeira.

PALAVRAS-CHAVE: Insetos sugadores, joaninha, pesticidas, café, seletividade, controlebiológico.

\section{ABSTRACT}

PHYSIOLOGIC SELECTIVITY OF PESTICIDES USED ON COFFEE PLANTATIONS ON EGGS AND ADULTS OF CRYPTOLAEMUS MONTROUZIERI MULSANT. On coffee plantations, the association between selective compounds and natural enemies composes an important tool for the pest management. The objective of this study was to evaluate some pesticides used on coffee plantations in regard to their lethal and sublethal effects on eggs and adults of Cryptolaemus montrouzieri Mulsant under laboratory conditions. The bioassays were carried out in the Laboratory of Selectivity Studies, Department of Entomology of the Universidade Federal de Lavras - UFLA, Brazil, under controlled conditions (climatic chamber) at $25 \pm 2^{\circ} \mathrm{C}, \mathrm{RH}$ of $70 \pm 10 \%$ with a $12 \mathrm{~h}$ photophase. The pesticides and doses in $\mathrm{g}$ or $\mathrm{mL}$ of i.a. $\mathrm{L}^{-1}$ were: thiamethoxam $(0.5)$, imidacloprid (0.7), mineral oil (13.3), endosulfan (2.63) and dimethoate (0.48). Distilled water was used as a control. The sprayings of the pesticides were accomplished using a Potter's tower. The parameters evaluated were: specimen survival after the application of the compounds, oviposition and eggs viability. Thiamethoxam, imidacloprid and endosulfan were the most harmful to the treated individuals. When sprayed on eggs, thiamethoxam increased the embryonic period length (7.1 days). Mineral oil was the only compound that did not affect the reproduction of C. montrouzieri. In function of the selectivity presented by mineral oil, it can be recommended to be used in integrated pest management on coffee plantations in association with this predator.

KEY WORDS: Sucking insects, ladybeetles, pesticides, coffee, selectivity, biological control.

\footnotetext{
${ }^{2}$ Universidade Federal de Lavras, Departamento de Entomologia, Lavras, MG, Brasil.

${ }^{3}$ Universidade Federal Rural do Rio de Janeiro, Departamento de Biologia, Seropédica, RJ, Brasil.

${ }^{4}$ Universidade Estadual Paulista, Faculdade de Ciências Agronômicas, Botucatu, SP, Brasil.

${ }^{5}$ UNIFENAS, Universidade "José do Rosário Vellano", Alfenas, MG, Brasil

*Mestranda em Agronomia/Entomologia, DEN/UFLA.

** Mestrando em Sistema de Produção na Agropecuária, UNIFENAS.

***Mestranda em Proteção de Plantas, FCA/UNESP.
} 


\section{INTRODUÇÃO}

A agricultura brasileira representa atualmente um dos principais pilares de divisas econômicas para o país. Neste cenário a cultura cafeeira figura como uma das de maior relevância na geração de divisas que fortalecem a balança comercial. A produção brasileira concentra-se nos estados de Minas Gerais, Espírito Santo e São Paulo (MAPA, 2007).

Apesar do grande sucesso da cultura cafeeira em solos nacionais, vários problemas podem afetar o desempenho dos cafezais, reduzindo a produtividade e a qualidade nas principais áreas cultivadas, entre os quais, pode-se citar a ocorrência de pragas. Ácaros, coleóperos, lepidópteros e hemípteros são freqüentes nas lavouras.

As espécies cochonilha-branca Planococcus citri (Risso) (Hemiptera: Pseudococcidae), cochonilhabranca Planococcus minor (Maskell) (Hemiptera: Pseudococcidae) e cochonilha-branca-de-cauda-longa Pseudococcus longispinus (Targioni Tozzetti) (Hemiptera: Pseudococcidae) são as cochonilhas-farinhentas mais comuns na parte aérea dos cafeeiros, sendo que suas ninfas ou fêmeas adultas sugam a seiva nas rosetas, resultando no chochamento e queda de botões florais efrutos ainda em desenvolvimento (SANTA-CECília et al., 2007).

O controle dessas pragas vem sendo realizado de formas variadas, entretanto, tem crescido no país o emprego do manejo integrado de pragas (MIP) nesta cultura, principalmente em sistemas de Produção Integrada de Café (PIC) que se encontra em fase de implantação no território brasileiro (BoLleretal.,1999; ANDRIGUETO; KOSOSKI, 2002; RAIJ, 2003; Boller et al., 2004). Nesse sistema preconiza-se, entre outras práticas de proteção ao meio ambiente, a redução no uso de produtos fitossanitários e intensificação do emprego de outros métodos para o controle de pragas, visando à obtenção de produtos de melhor qualidade. Desta forma, o uso de inimigos naturais, como predadores e/ ou parasitóides está sendo bastante incentivado.

Destaca-se, dentre o grupo de inimigos naturais que habitam o agroecossistema cafeeiro, os coccinelídeos (Coleoptera: Coccinellidae), conhecidos comojoaninhas, organismos eficientes no controle de vários insetos-praga. Existem cerca de 4.200 espécies pertencentes a esta família, das quais aproximadamente 90\% são predadoras (СНАСКО et al., 1978; IPERTI, 1999). Pertencente a esta ordem, a espécie Cryptolaemus montrouzieri Mulsant é a mais eficiente no controle de cochonilhas, principalmente do gênero Planococcus sp. É uma espécie nativa da Austrália e foi o primeiro agente biológico introduzidona Índia, em 1898, para o controle de Coccus viridis (Green) em cafeeiro (Puttarudriah et al., 1952, citado por СНАско et al., 1978), sendo posteriormente empregada na Califórnia (EUA) para o controle de P. citri em citros (Smith; Armitage, 1920).

Em literaturas, nacional e internacional, poucos são os trabalhos recentes relacionados ao efeito de pesticidas sobre C.montrouzieri, entretanto, até 1990 era considerado $015^{\circ}$ inimigo natural mais estudado em termos de seletividade (СRоғт, 1990). СНАско et al.(1978) já relataram a importância de se conhecer o efeito dos pesticidas sobre esse predador, como forma de garantir o sucesso do controle biológico. No Brasil, o presente estudo é o primeiro a ser realizado.

Avaliações dos efeitos de acaricidas, inseticidas e fungicidas na mortalidade e efeitos subletais sobre $C$. montrouzieri já realizadas, evidenciaram tolerância diferenciada desse predador aos diversos produtos fitossanitários (Bellows et al., 1985; Morse; Bellows, 1986; Morse et al., 1987; Babu; Azam, 1987; Bellows; Morse, 1988; MANi et al., 1997; BOYero et al., 2005; Cloyd; Dickinson, 2006). Tais efeitos podem ainda interferir na compatibilização do emprego de inimigos naturais em programas de MIP ou PIC em condições de campo.

Desta maneira, considerando-se a potencialidade dos predadores da espécie C. montrouzieri no controle de cochonilhas em cafeeiros e a necessidade da compatibilização desses organismos com aplicações de pesticidas, o objetivo do trabalho foi avaliar a seletividade fisiológica de inseticidas utilizados na cultura cafeeira sobre ovos e adultos desse predador.

\section{MATERIAL E MÉTODOS}

\section{Realização dos bioensaios em laboratório}

Os bioensaios seguiram a metodologia proposta por membros da Organização Internacional para o Controle Biológico (IobC/WPRS, 1992; JANSEN;H HaUtier, 2005). Os inseticidas utilizados e suas respectivas dosagens de aplicação, em $\mathrm{g}$ ou $\mathrm{mL}$ de i.a. $\mathrm{L}^{-1}$ foram: tiametoxam $(0,5)$, imidacloprido $(0,7)$, óleo mineral $(13,3)$, endossulfam $(2,63)$ e dimetoato $(0,48)$. A testemunha foi composta apenas por água destilada. A aplicação dos produtos foi realizada por meio de torre de Potter.

Criação de manutenção de C. montrouzieri em laboratório

Paraimplantação da criação de manutenção, adultos de C. montrouzieri provenientes da empresa Gravena Manecol foram acondicionados em de placas de Petri de $15 \mathrm{~cm}$ de diâmetro, fechadas com filme de PVC transparente e mantidas em câmaras 
climatizadas reguladas a $25 \pm 2{ }^{\circ} \mathrm{C}$, UR de $70 \pm 10 \% \mathrm{e}$ fotofase de 12 horas. Os predadores foram alimentados com uma massa cotonosa contendo ovos, ninfas e adultos de P. citri, provenientes de uma criação de laboratório e para o fornecimento deágua foi disposto um chumaço de algodão hidrófilo umedecido no interior da placa.

As posturas do predador em massas de ovos da cochonilha foram periodicamente removidas e transferidas para novas placas, para desenvolvimento das fases de larva, pupa e adulta. Após a emergência dos adultos, estes foram separados em novas placas de Petri para dar início a um novo ciclo de desenvolvimento.

\section{Efeito dos inseticidas sobre ovos deC.montrouzieri}

Ovos com aproximadamente 12 horas de idade foram retirados da criação de laboratório e colocados em placas de Petri de $15 \mathrm{~cm}$ de diâmetro. As pulverizações dos compostos foram realizadas diretamente sobre os ovos por meio de torre de Potter, com a aplicação de 1,5 $\pm 0,5 \mathrm{mg} \cdot \mathrm{cm}^{-2}$.

Após os ovos receberem os produtos, foram individualizados em tubos de vidro de $2,5 \mathrm{~cm}$ de diâmetro $\times 8,5 \mathrm{~cm}$ de altura, vedados com filme de PVC transparente e mantidos em câmara climatizada a $25 \pm 2{ }^{\circ} \mathrm{C}$, UR de $70 \pm 10 \%$ e 12 horas de fotofase. As larvas eclodidas e sobreviventes foram alimentadasadlibitum, a cada dois dias, com massa de ovos, ninfas e adultos da cochonilha $P$. citri, até a fase adulta.

O delineamento foi o inteiramente ao acaso, com seis tratamentos e dez repetições, sendo cada parcela composta por três ovos, totalizando 30 ovos/tratamento. Avaliaram-se o número de ovos viáveis e a duração do período embrionário.

Efeito dos inseticidas sobre adultos de $C$. montrouzieri

Adultos com 24 a 36 horas de idade foram separados por sexo e colocados em placas de Petri forradas com papel-filtro, onde receberam os produtos em pulverização, conforme metodologia utilizada na pulverização de ovos e larvas. Posteriormente, um casal por repetição, foi transferido para placa de Petri de $15 \mathrm{~cm}$ de diâmetro forrada com papel-filtro e vedada na parte superior com filme de PVC transparente. Os adultos foram alimentados ad libitum com a cochonilha P. citri. Foi utilizado o delineamento experimental inteiramente ao acaso, com seis tratamentos e 10 repetições, sendo cada unidade experimental constituída por dois espécimes. As avaliações da mortalidade de adultos foram realizadas $1 \mathrm{~h}, 1,2,3,4$ e 5 dias após as pulverizações.
Efeito dos produtos sobre os parâmetros reprodutivos de $C$. montrouzieri

Avaliaram-se o período de pré-oviposição, fecundidade das fêmeas e a viabilidade dos ovos colocados durante os primeiros 20 dias de oviposição. Espécimes oriundos de ovos ou adultos tratados, foram separados em casais e acondicionados em placas de Petri de $5 \mathrm{~cm}$ de diâmetro e fechadas com filme de PVC transparente. Os espécimes foram alimentados ad libitum por meio do fornecimento de água (em algodão umedecido) eovos, ninfas e adultos da cochonilha P.citri. Para estudos da viabilidade dos ovos, foram coletados ao acaso 30 ovos de cada tratamento a cada semana por um período de vinte dias.

Para os testes foi utilizado o delineamento experimental inteiramente ao acaso, com número variável de tratamentos e 7 repetições, cada uma composta por um casal de indivíduos. O número de tratamentos e repetições foi variável em função da mortalidade provocada pelo produto.

\section{Análise estatística}

Os dados de sobrevivência após 1h, 1,2,3,4e 5 dias após a aplicação dos produtos sobre adultos foram transformados para arcoseno $\sqrt{(x / 100)}$ e submetidos à análise de variância em um modelo de parcelas subdivididas no tempo, com os produtos na parcela.

As médias de sobrevivência dos espécimes nos instares subseqüentes à aplicação sobre ovos. Utilizou-se um esquema fatorial de $6 \times 4$ em um delineamento inteiramenteao acaso de produtos $x$ número deinstares com quatro 4 repetições, utilizando-se cinco insetos em cada unidade experimental (quando possível).

Ascomparações das médias dos tratamentosforam realizadas por meio do teste de Scott e Knott a 5\% de significância (SсOTT; KNOTT, 1974). O mesmo modelo de análise foiadotado para os dados referentes aos efeitos dos compostos sobre os parâmetros reprodutivos dos insetos tratados nas diferentes fases de desenvolvimento. Para os dados balanceados, foi utilizado o programa estatístico Sisvar (FERREIRA, 2000). Quando o número derepetições foi diferente para os tratamentos, utilizou-se o proc GLM do SAS (SAS INSTITUTE, 2001).

\section{Classificação dos produtos quanto à toxicidade segundo escala da IOBC}

Os produtos foram enquadrados em classes de toxicidade conforme as recomendações sugeridas por membros da IOBC (VEIRE et al., 2002), onde: classe 1 = inócuo $(<30 \%)$, classe 2 = levemente nocivo $(30 \% \leq \mathrm{E}$ $\leq 80 \%)$, classe $3=$ moderadamentenocivo $(80<\mathrm{E} \leq 99 \%)$ e classe $4=$ nocivo ( $>99 \%$ de mortalidade), em função do efeito sobre os parâmetros reprodutivos e mortali- 
dade do predador, sendo o efeito total (E\%) calculado pela fórmula proposta por VoGT (1992):

$$
E=100 \%-(100 \%-M \%) \times R_{1} \times R_{2}
$$

$\mathrm{Na}$ qual: $\mathrm{E}=$ efeito total $(\%) ; \mathrm{M} \%=$ mortalidade total, corrigida em função do tratamento testemunha (Аввотт, 1925); $R_{1}=$ razão entre a média diária de ovos colocados por fêmea tratada e não tratada; $R_{2}=$ razão entre a média de ovos viáveis colocados por fêmea tratada e não tratada.

\section{RESULTADOS E DISCUSSÃO}

\section{Efeito dos inseticidas sobre a viabilidade de ovos}

Tiametoxam, imidacloprido e dimetoato causaram a mortalidade de embriões do predador, levando a uma redução significativa nas porcentagens de larvas eclodidas (Tabela 1). Observou-se também que tiametoxam prolongou o desenvolvimento de embriões e larvas provenientes de ovos tratados e reduziu ainda a viabilidade da fase larval (Tabela 1).

Desde o início da segunda metade do século passado, trabalhos como o de Ripper et al. (1951) têm buscado compreender o efeito de pesticidas sobre os inimigos naturais. Entretanto, nos últimos anos as pesquisas têm dado ênfase não só para a mortalidade de espécimes adultos, mas também às diferentes fases de desenvolvimento e ainda aos efeitos subletais que os compostos podem provocar nos organismos nãoalvo, como apresentado no presente estudo.

Conforme os resultados obtidos para ovos e larvas, verificou-se que os inseticidas apresentaram comportamentos distintos. Tiametoxam e imidacloprido foram mais severos, dimetoato e endossulfam, tiveram, de modo geral, efeitos intermediários e o óleo mineral apresentou-se como menos prejudicial.

Tiametoxam e imidacloprido são compostos pertencentes ao grupo dos neonicotinóides e atuam por contato e ingestão, apresentando também ação translaminar e atividade sistêmica em tecidos de plantas (PMrA, 2001). Tais propriedades podem estar relacionadas ao fato dos produtos terem penetrado no interior dos ovos e provocado efeito tóxico sobre os embriões.

Os compostos neonicotinóides são potentes agonistas da acetilcolina nas junções colinérgicas do sistema nervoso dos insetos e como não são degradados pela acetilcolinesterase, provocam a morte dos insetos (WARE; WHITACRE, 2004). Esta ação pode ter sido conferida às larvas de C. montrouzieri de forma severa quando expostas aos produtos tiametoxam e imidacloprido, ocasionando a sua morte.

Efeito dos compostos sobre os parâmetros reprodutivos de fêmeas de $C$. montrouzieri tratadas nas fases de ovo

Os compostos não afetaram o período de préoviposição, com médias que variaram de 4,8 a 5,9 dias; todavia, endossulfam e dimetoato foram prejudiciais aos parâmetros números de ovos colocados por fêmeas do predador oriundas de ovos tratados (diário e total). A viabilidade dos ovos colocados não foi afetada por nenhum produto avaliado. Para tiametoxam e imidacloprido não foi possível avaliar tais características biológicas em razão da reduzida taxa de eclosão de larvas, uma vez que os compostos provocaram elevada mortalidade (Tabela 2).

Em insetos, poucos são os trabalhos disponíveis em literatura que relatam efeitos de dimetoato sobre a reprodução. Sobre Collembola, Petersen; Gelstrup (1998) verificaram que além de afetar o crescimento e pesos dos insetos, também reduziu a capacidade reprodutiva de espécimes de Folsomia fimetaria (Linnaeus) (Collembola: Isotomidae) e Hypogastrura assimilis Krausbauer (Collembola: Hypogastruridae) avaliadas.

Tabela 1 - Viabilidade de ovos tratados (\%), duração do período embrionário (dias), duração da fase larval (dias) e viabilidade da fase larval (\%) $( \pm \mathrm{EP})$ de espécimes de Cryptolaemus montrouzieri, submetidos ao tratamento na fase de ovo. Temperatura de $25 \pm 2^{\circ} \mathrm{C}$, UR de $70 \pm 10 \%$ e fotofase de 12 horas.

\begin{tabular}{lcccc}
\hline Tratamentos & $\begin{array}{c}\text { Viabilidade de } \\
\text { ovos tratados }\end{array}$ & $\begin{array}{c}\text { Duração do período } \\
\text { embrionário }\end{array}$ & $\begin{array}{c}\text { Duração da } \\
\text { fase larval }\end{array}$ & $\begin{array}{c}\text { Viabilidade da } \\
\text { fase larval }\end{array}$ \\
\hline Tiametoxam & $26,6 \pm 4,08 \mathrm{c}$ & $7,1 \pm 3,45 \mathrm{~b}$ & $29,3 \pm 5,35 \mathrm{~b}$ & $13,3 \pm 2,25 \mathrm{~b}$ \\
Imidacloprido & $13,3 \pm 7,42 \mathrm{c}$ & $6,5 \pm 0,08 \mathrm{a}$ & - & - \\
Óleo mineral & $80,0 \pm 2,18 \mathrm{a}$ & $5,3 \pm 1,15 \mathrm{a}$ & $24,8 \pm 2,39 \mathrm{a}$ & $80,0 \pm 5,93 \mathrm{a}$ \\
Endossulfam & $91,3 \pm 3,12 \mathrm{a}$ & $5,1 \pm 0,04 \mathrm{a}$ & $23,9 \pm 3,86 \mathrm{a}$ & $93,3 \pm 6,28 \mathrm{a}$ \\
Dimetoato & $66,6 \pm 6,45 \mathrm{~b}$ & $6,1 \pm 0,50 \mathrm{a}$ & $23,9 \pm 5,12 \mathrm{a}$ & $66,6 \pm 4,49 \mathrm{a}$ \\
Testemunha & $100,0 \pm 0,00 \mathrm{a}$ & $5,4 \pm 1,59 \mathrm{a}$ & $25,3 \pm 2,19 \mathrm{a}$ & $86,7 \pm 7,55 \mathrm{a}$ \\
\hline $\mathrm{CV}(\%)$ & 17,79 & 22,29 & 31,65 & 26,78 \\
\hline
\end{tabular}

Médias seguidas pela mesma letra na coluna não diferem estatisticamente entre si pelo teste de Scott e Knott $(P<0,05)$.

(-) Não foi possível avaliar o parâmetro biológico em função da alta mortalidade provocada pelo produto. 
Tabela 2 - Período de pré-oviposição (dias), número médio diário e total de ovos colocados em 20 dias e a viabilidade $( \pm \mathrm{EP})$, obtidos de fêmeas de Cryptolaemus montrouzieri provenientes de ovos tratados com os produtos. Temperatura de $25 \pm 2{ }^{\circ} \mathrm{C}$, UR de $70 \pm 10 \%$ e fotofase de 12 horas.

\begin{tabular}{lcccc}
\hline Tratamentos & Pré-oviposição (dias) & $\begin{array}{c}\text { No médio } \\
\text { diário de ovos }\end{array}$ & Total de ovos/20 dias & Viabilidade dos ovos (\%) \\
\hline Tiametoxam & - & - & - & - \\
Imidacloprido & - & - & - & - \\
Óleo mineral & $5,3 \pm 0,58 \mathrm{a}$ & $3,9 \pm 1,50 \mathrm{a}$ & $78,8 \pm 9,58 \mathrm{a}$ & $85,6 \pm 9,46 \mathrm{a}$ \\
Endossulfam & $4,8 \pm 1,96 \mathrm{a}$ & $2,3 \pm 1,16 \mathrm{~b}$ & $46,2 \pm 8,26 \mathrm{~b}$ & $82,3 \pm 5,36 \mathrm{a}$ \\
Dimetoato & $5,8 \pm 2,11 \mathrm{a}$ & $2,4 \pm 1,01 \mathrm{~b}$ & $49,0 \pm 8,59 \mathrm{~b}$ & $81,6 \pm 5,09 \mathrm{a}$ \\
Testemunha & $5,9 \pm 0,94 \mathrm{a}$ & $4,2 \pm 1,12 \mathrm{a}$ & $84,8 \pm 6,93 \mathrm{a}$ & $80,6 \pm 4,38 \mathrm{a}$ \\
\hline CV $(\%)$ & 26,13 & 29,27 & 24,30 & 13,44
\end{tabular}

Médias seguidas pela mesma letra na coluna não diferem estatisticamente entre si pelo teste de Scott e Knott $(\mathrm{P}<0,05)$.

(-) Não foi possível formar casais, em função da alta mortalidade provocada pelo produto.

Os resultados do presente estudo indicam a possibilidade do dimetoato ter afetadoa espermatogênese ou a oogênese durante o período de maturação do sistema reprodutivo de C. montrouzieri, ocasionando redução na fecundidade dos ovos, assim como foi verificado por Petersen; GJelstrup (1998).

Para endossulfam, o efeito conferido sobre os parâmetros reprodutivos de fêmeas tratadas nas fases de ovo e quarto instar pode ter ocorrido pelo fator de bioacumulação do composto nos tecidos dos insetos. De acordo com dados do arquivo ExTOXNET (1996) e Ware; Whitacre (2004) o composto pertence ao grupo químico dos organoclorados e apresenta alto potencial para bioacumulação nos tecidos gordurosos de artrópodes. Ovos de C. montrouzieri podem apresentar maiores teores de gorduras em seus tecidos de reserva, facilitando o seqüestro do pesticida no organismo. Com a retenção do composto, sua ação pode ter sido revelada na fase reprodutiva, em função do elevado consumo de energia pelos insetos.

\section{Efeito dos produtos fitossanitários sobre adultos} de C. montrouzieri

\section{Na sobrevivência}

A fase adulta também se mostrou bastante sensível aos pesticidas tiametoxam, imidacloprido, endossulfam e dimetoato, que resultaram em altos valores de mortalidade.

Uma hora após a aplicação dos produtos, verificou-sequeimidacloprido apresentou efeito tóxico aos adultos de C. montrouzieri, com média de $46,7 \%$ de sobrevivência. Decorridas 24 horas desde a pulverização com tiametoxam, imidacloprido e dimetoato observou-se redução significativa na sobrevivência dos espécimes, com médias de 13,3\%;33,3\% e 33,3\%, respectivamente e ainda, não se observaram sobreviventes tratados com endossulfam (Tabela3). No quinto dia após a pulverização, os produtos tiametoxam, imidacloprido, óleo mineral, endossulfam e dimetoato apresentaram médias de sobrevivência de 6,7\%;9,0\% ; $80,0 \% ; 0,0 \%$ e $26,6 \%$, respectivamente.

Para o óleo mineral constatou-se que após 24 horas desdea aplicaçãoocorreu uma reduçãona sobrevivência dos espécimes, chegando a $80 \%$ ao final da avaliação.

Estudando a toxicidade direta de óleo mineral (20

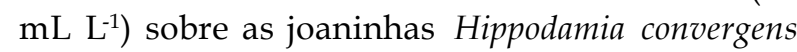
Guérin-Ménéville (Coleoptera: Coccinellidae), Coleomegilla maculata (DeGeer) (Coleoptera: Coccinellidae), Harmonia axyridis (Pallas) e $C$. montrouzieri, SMITH; KRISCHIK (2000) também não observaram efeito tóxico do composto na sobrevivência desses predadores e classificaram o composto como seletivo para os predadores.

\section{Na reprodução}

A alta mortalidade provocada por tiametoxam, imidacloprido e endossulfam aos adultos de $C$. montrouzieri tratados impossibilitou a avaliação dos seus efeitos sobre as características reprodutivas desse predador. Verificou-se que óleo mineral não afetou o período de pré-oviposição, número médio de ovos colocados diariamente e tampouco o total de ovos colocados em 20 dias de avaliação; entretanto, fêmeas tratadas com dimetoato apresentaram redução no número médio diário de ovos e também no total de ovos colocados (Tabela4). Operíodo de pré-oviposição e a viabilidade dos ovos não foram afetados por nenhum dos compostos submetidos à avaliação.

\section{Efeito total dos produtos fitossanitários sobre o predador de acordo com escala proposta pela IOBC}

A alta mortalidade apresentada pelos compostos neonicotinóides resultou na classificação desses compostos como altamente prejudiciais ao predador, sendo enquadrados na classe 4 para ovos e adultos (Tabela 5). 
Tabela 3 -Sobrevivência (\%) ( \pm EP) de adultos de Cryptolaemus montrouzieriapós a aplicação dos produtos fitossanitários. Temperatura de $25 \pm 2^{\circ} \mathrm{C}$, UR de $70 \pm 10 \%$ e fotofase de 12 horas.

\begin{tabular}{lrrrrrr}
\hline Tratamentos & \multicolumn{5}{c}{ Sobrevivência (\%)/dias após aplicação } \\
\cline { 2 - 7 } & \multicolumn{1}{c}{1 hora } & \multicolumn{1}{c}{1 dia } & \multicolumn{1}{c}{2 dias } & \multicolumn{1}{c}{3 dias } & 4 dias & 5 dias \\
\hline Tiametoxam & $86,7 \pm 2,25 \mathrm{aA}$ & $13,3 \pm 2,41 \mathrm{bB}$ & $6,7 \pm 1,33 \mathrm{~dB}$ & $6,7 \pm 1,33 \mathrm{~dB}$ & $6,7 \pm 1,33 \mathrm{~dB}$ & $6,7 \pm 1,33 \mathrm{cB}$ \\
Imidacloprido & $46,7 \pm 6,41 \mathrm{bA}$ & $33,3 \pm 3,97 \mathrm{bB}$ & $20,0 \pm 3,27 \mathrm{cC}$ & $20,0 \pm 3,27 \mathrm{cC}$ & $13,3 \pm 3,01 \mathrm{dC}$ & $9,0 \pm 3,01 \mathrm{cC}$ \\
Óleo mineral & $100,0 \pm 0,00 \mathrm{aA}$ & $86,7 \pm 3,29 \mathrm{aB}$ & $80,0 \pm 4,96 \mathrm{bB}$ & $80,0 \pm 4,96 \mathrm{bB}$ & $80,0 \pm 4,96 \mathrm{bB}$ & $80,0 \pm 4,96 \mathrm{aB}$ \\
Endossulfam & $100,0 \pm 0,00 \mathrm{aA}$ & $0,0 \pm 0,00 \mathrm{cB}$ & - & - & - & - \\
Dimetoato & $100,0 \pm 0,00 \mathrm{aA}$ & $33,3 \pm 4,23 \mathrm{bB}$ & $26,6 \pm 3,46 \mathrm{cB}$ & $26,6 \pm 3,46 \mathrm{cB}$ & $26,6 \pm 3,46 \mathrm{cB}$ & $26,6 \pm 3,46 \mathrm{bB}$ \\
Testemunha & $100,0 \pm 0,00 \mathrm{aA}$ & $100,0 \pm 0,00 \mathrm{aA}$ & $100,0 \pm 0,00 \mathrm{aA}$ & $100,0 \pm 0,00 \mathrm{aA}$ & $100,0 \pm 0,00 \mathrm{aA}$ & $93,3 \pm 5,54 \mathrm{aA}$ \\
\hline
\end{tabular}

$\mathrm{CV}(\%)$ 23,28

Médias seguidas pela mesma letra, minúsculas na coluna e maiúsculas na linha não diferem estatisticamente entre si pelo teste de Scott e Knott (P < 0,05). (-) O composto provocou mortalidade de $100 \%$ dos espécimes tratados.

A exposição de ovos do predador ao endossulfam e dimetoato causou baixo efeito tóxico e os compostos foram considerados levemente nocivos (classe 2). $\mathrm{O}$ óleo mineral não apresentou ação ovicida e causou apenas 9,0\% de efeito total sobre os espécimes (classe 1) (Tabela 5).

Com exceção do óleo mineral, todos os produtos testados foram prejudiciais aos predadores, quando pulverizados sobre os insetos na fase adulta. Os inseticidas tiametoxam, imidaclopridoe endossulfam foram nocivos e enquadrados na classe 4, apresentando valores de efeito total aos predadores da ordem de $100 \%$ (Tabela 5). Dimetoato foi categorizado como moderadamente nocivo e enquadrado na classe 3, com uma média de efeito total de $81,4 \%$.

Óleo mineral não apresentou efeito tóxico significativo, com efeito total de cerca de $29,7 \%$, sendoenquadrado na classe 1 , sendo considerado inócuo (Tabela 5).

Os estudos já realizados e disponíveis em literatura relataram que adultos de C. montrouzieri apresen- tam respostas variadas aos compostos químicos, e que a maioria das populações é mais suscetível aos compostos neurotóxicos (MORSE;B Ellows, 1986;MORSE et al., 1987; RAMESH; AZAM, 1987; CASTAÑER; GARRIDO, 1995; Mani et al., 1997; Boyero et al., 2005; Cloyd; DiCKINSON, 2006).

Dentre os compostos neurotóxicos, os organofosforados apresentam alto impacto sobre os coccinelídeos. Em dezessete compostos deste grupo já testados, a média de toxicidade calculada variou de 2,8 a 3,0 na escala proposta pela IOBC que varia de 1 até 4 (Miret; GARCiA-MARI, 2001). Em ensaios realizados por Boyeroet al. (2005) os compostos organofosforados metidatiom e malatiom causaram elevada mortalidade de C. montrouzieri 24 horas após a sua aplicação. Esses resultados assemelham-se aos obtidos neste estudo para dimetoato, onde 24 horas após a sua pulverização, a mortalidade dessa espécie de predador foi de cerca de $70 \%$.

Tabela 4 - Período de pré-oviposição (dias), número médio diário e total de ovos colocados em 20 dias e a viabilidade $( \pm \mathrm{EP})$, obtidos de espécimes de Cryptolaemus montrouzieri provenientes de adultos tratados com os produtos. Temperatura de $25 \pm 2^{\circ}$ C, UR de $70 \pm 10 \%$ e fotofase de 12 horas.

\begin{tabular}{lcccc}
\hline Tratamentos & Pré-oviposição (dias) & № médio diário de ovos & Total de ovos/ 20 dias & $\begin{array}{c}\text { Viabilidade dos } \\
\text { ovos }(\%)\end{array}$ \\
\hline Tiametoxam & - & - & - & - \\
Imidacloprido & - & - & - & - \\
Óleo mineral & $6,0 \pm 1,32 \mathrm{a}$ & $2,9 \pm 0,50 \mathrm{a}$ & $57,0 \pm 5,77 \mathrm{a}$ & $75,6 \pm 7,35 \mathrm{a}$ \\
Endossulfam & - & - & - & - \\
Dimetoato & $5,8 \pm 1,96 \mathrm{a}$ & $2,1 \pm 0,99 \mathrm{~b}$ & $41,0 \pm 4,47 \mathrm{~b}$ & $83,4 \pm 9,38 \mathrm{a}$ \\
Testemunha & $5,3 \pm 0,94 \mathrm{a}$ & $3,2 \pm 0,48 \mathrm{a}$ & $63,2 \pm 4,00 \mathrm{a}$ & $83,5 \pm 6,35 \mathrm{a}$ \\
\hline CV $(\%)$ & 9,22 & 12,64 & 23,49 & 27,31
\end{tabular}

Médias seguidas pela mesma letra na coluna não diferem estatisticamente entre si pelo teste de Scott e Knott $(\mathrm{P}<0,05)$.

(-) Não foi possível formar casais, em função da alta mortalidade provocada pelo produto. 
Tabela 5 - Mortalidade (\%), oviposição, efeito total (E\%) e classificação dos produtos em função da escala de toxicidade proposta pela IOBC, de espécimes oriundos de pupas e adultos de Cryptolaemus montrouzieritratados. Temperatura de $25 \pm 2^{\circ} \mathrm{C}$, UR de $70 \pm 10 \%$ e fotofase de 12 horas.

\begin{tabular}{|c|c|c|c|c|c|c|c|}
\hline Tratamentos & № casais & Mortalidade & $\mathrm{R}_{1}^{1}$ & $\mathrm{R}_{2}^{2}$ & $\mathrm{M} \%^{3}$ & $\mathrm{E}(\%)^{4}$ & Classe $^{5}$ \\
\hline & \multicolumn{7}{|c|}{ Ovos tratados } \\
\hline Tiametoxam & - & 83,7 & - & - & 81,2 & 100,0 & 4 \\
\hline Imidacloprido & - & 100,0 & - & - & 100,0 & 100,0 & 4 \\
\hline Óleo mineral & 7 & 20,0 & 3,9 & 85,6 & 7,7 & 9,0 & 1 \\
\hline Endossulfam & 7 & 8,7 & 2,3 & 82,3 & 0,0 & 43,9 & 2 \\
\hline Dimetoato & 7 & 33,4 & 2,4 & 81,6 & 23,2 & 55,8 & 2 \\
\hline \multirow[t]{2}{*}{ Testemunha } & 7 & 13,3 & 4,2 & 80,6 & - & - & - \\
\hline & \multicolumn{7}{|c|}{ Adultos tratados } \\
\hline Tiametoxam & - & 93,3 & - & - & 92,8 & 100,0 & 4 \\
\hline Imidacloprido & - & 90,0 & - & - & 89,6 & 100,0 & 4 \\
\hline Óleo mineral & 7 & 20,0 & 2,9 & 75,6 & 14,3 & 29,7 & 1 \\
\hline Endossulfam & - & 100,0 & - & - & 100,0 & 100,0 & 4 \\
\hline Dimetoato & 2 & 73,4 & 2,1 & 83,4 & 71,4 & 81,4 & 3 \\
\hline Testemunha & 7 & 6,7 & 3,2 & 83,5 & - & - & - \\
\hline
\end{tabular}

${ }^{1}$ Oviposição média/fêmea/dia.

${ }^{2}$ Viabilidade de ovos (\%).

${ }^{3}$ Mortalidade no tratamento corrigida pela fórmula de Аввотт (1925).

${ }^{4}$ Efeito total do produto sobre o predador. $E=100 \%-(100 \%-M \%) \times R_{1} \times R_{2}$.

${ }^{5}$ Classe de toxicidade: classe $1=$ inócuo $(\mathrm{E}<30 \%)$, classe $2=$ levemente nocivo $(30 \% \leq \mathrm{E} \leq 80 \%)$, classe $3=$ moderadamente nocivo $(80 \%<\mathrm{E} \leq 99 \%)$ e classe $4=$ nocivo $(\mathrm{E}>99 \%)$ (VEIRE et al., 2002).

A ação direta e indireta de neonicotinóides sobre C. montrouzieri foi estudada por CloYD; DiCKINSON (2006) cujos resultados evidenciaram mortalidade elevada, 24 horas após a aplicação dos compostos. Decorridas 48 horas, a mortalidade atingiu 100\% para os tratamentos à base de acetamiprido e clotianidino (enquadrados na classe 4). Esses pesticidas pertencem ao mesmo grupo químico de imidacloprido e tiametoxam, avaliados neste estudo, com resultados semelhantes.

Na literatura, são escassos os estudos que abordam os efeitos de tiametoxam sobre esse predador e, em função do seu efeito prejudicial constatado no presente trabalho, novas pesquisas deverão ser realizadas para confirmar ou não a sua toxicidade para este inimigo natural em condições de semicampo e campo. É importante ressaltar ainda, que este composto é recomendado para aplicação via solo na cultura do café, podendo esta forma de aplicação, conferir seletividade fisiológica para alguns inimigos naturais presentes no agroecossistema cafeeiro.

\section{CONCLUSÕES}

Os pesticidas tiametoxam e imidacloprido são nocivos paraC.montrouzierinas fases de ovo eadulta, quando aplicado sobre os insetos.
Emdossulfamé nocivo paraC. montrouzieri quando aplicado sobre adultos, eélevemente nocivo para ovos.

Dimetoato é levemente nocivo para ovos e moderadamente nocivo para e adultos de C. montrouzieri.

Óleo mineralé pouco prejudicial paraC.montrouzieri e, portanto, pode ser empregado em programas de manejo integrado de cochonilhas da parte aérea de cafeeiros, visando à preservação desse predador.

\section{AGRADECIMENTOS}

Os autores agradecemo apoio do À Coordenadoria de Aperfeiçoamento de Pessoal de Ensino Superior (Capes), eao Conselho Nacional de Desenvolvimento Científico e Tecnológico (CNPq).

À Empresa Brasileira de Pesquisa Agropecuária (Embrapa Café) e ao Consórcio Brasileiro de Pesquisa eDesenvolvimento doCafé(CBP\&D-Café), peloaporte financeiro e esta pesquisa.

À Fundação de Amparo à Pesquisa do Estado de Minas Gerais (FAPEMIG) pelo aporte financeiro para a realização do projeto de pesquisa.

\section{REFERÊNCIAS}

ABBOTT, W.S. A method of computing the effectiveness of an insecticide. Journal of Economic Entomology, v.18, n.2, p.265-267, 1925. 
ANDRIGUETO, J.R.; KOSOSKI, A.R. Marco legal da produção integrada de frutas no Brasil. Brasília: MAPA/ SARC, 2002. 60p.

BABU, T.R.; AZAM, K.M. Residual toxicity of different insecticides to the adult Cryptolaemus montrouzieri Mulsant (Coccinellidae: Coleoptera). Tropical Pest Management, v.33, n.2, p.180-181, 1987.

BELLOWS, T.S.J.; MORSE, D.G. Residual toxicity following dilute or low-volume applications of insecticides used for control of California red scale (Homoptera: Diaspididae) to four beneficial species in citrus agroecosystem. Journal of Economic Entomology, v.81, v.3, p.892-898, 1988.

BELLOWS, T.S.J.; MORSE, D.G.; HADJIDEMETRIOU, D.G.; IWATA, Y. Residual toxicity of four insecticides used for control of citrus thrips (Thysanoptera: Thripidae) on three beneficial species in a citrus agroecosystem. Journal of Economic Entomology, v.78, p.681-686, 1985.

BOLLER, E.F.; AVILLA, J.; JOERG, E.; MALAVOLTA, C.; WIJNANDS, F.G.; ESBJERG, P. Integrated production: principles and technical guidelines. 3.ed. Wädenswil: IOBC/WPRS/OILB/SROP, 2004. v.27, 54p. (Bulletin IOBC/WPRS, OILB/SROP, 2).

BOLLER, E.F.; TITI, A.E.; GENDRIER, J.P.; AVILLA, J.; JOER, E.; MALAVOLTA, C. Integrated production: principles and technical guidelines. 2.ed. Wädenswil: IOBC/WPRS/OILB/SROP, 1999. v.22, 29p. (Bulletin IOBC/WPRS, OILB/SROP, 4).

BOYERO, J.R.; RODRÍGUEZ, N.; SURIA, R.; RUÍZ, R.; PASCUAL, F. Efectos de varios plaguicidas sobre Cryptolaemus montrouzieri Mulsant y Rhyzobius lophantae Blaisdell (Coleoptera: Coccinellidae). Boletín de Sanidad Vegetal de Plagas, v.31, n.1, p.79-87, 2005.

CASTAÑER, M.; GARRIDO, A. Toxicidad producida por contato y persistência de diversos plaguicidas sobre três insetos utilizados en control biologico: Cryptolaemus montrouzieri, Lysiphlebus testaceipes y Encarsia formosa. Investigaciones Agrarias: Producción y Protección Vegetales, v.10, n.1, p.139-147, 1995.

CHACKO, M.J.; BHAT, P.K.; RAO, L.V.A.; SINGH, M.B.D.; RAMANARAYAN, E.P.; SREEDHRAN, K. The use of the ladybird beetle Cryptolaemus montrouzieri, for the control of coffee mealybugs. Journal of Coffee Research, v.8, n.1, p.14-19, 1978.

CLOYD, R.A.; DICKINSON, A. Effect of insecticides on mealybug destroyer (Coleoptera: Coccinellidae) and parasitoid Leptomastix dactylopii (Hymenoptera: Encyrtidae), natural enemies of citrus mealybug (Homoptera: Pseudococcidae). Journal of Economic Entomology, v.99, n.5, p.1596-1604, 2006.
CROFT, B. A. Arthropod biological control agents and pesticides. Environmental Science and Technology. New York: Wiley-Interscience, 1990. 723p.

EXTOXNET - EXTENSION TOXICOLOGY NETWORK. Pesticides information profiles: endosulfan. Cornell, 1996. Disponível em: <http://extoxnet.orst.edu/pips/ endosulf.htm>. Acesso em: 27 nov. 2007.

FERREIRA, D.F. Análises estatísticas por meio do Sisvar para Windows versão 4.0. In: REUNIÃO ANUAL DA REGIÃO BRASILEIRA DA SOCIEDADE INTERNACIONAL DE BIOMETRIA, 45., 2000, São Carlos. Anais. São Carlos: UFSCar, 2000. p.255-258.

IOBC/WPRS. INTERNATIONAL ORGANIZATION FOR BIOLOGICAL CONTROL. West Palaearctic Regional Section. Working Group "Pesticides and Beneficial Organisms", Guidelines for testing the effects of pesticides on beneficial organisms: description of test methods. IOBCMPRS Bulletin, v.15, p.1-186. 1992.

IPERTI, G. Biodiversity of predaceous Coccinellidae in relation to bioindication and economic importance. Agriculture Ecosystems and Environments, v.74, n.1/3, p.323-342, 1999.

JANSEN, J.P.; HAUTIER, L. Comparative sensitivity of four ladybird species to five pesticides. In: VOGT, H.; OLSZAK, R.; GAJEK, D. IOBC/WPRS - Working Group Pesticides and Beneficial Organisms Poland: Meeting Dêbe, 2005. p.5-6.

MANI, M.; LAKSHIMI, V.J.; KRISHNAMOORTHY, A. Side effects of some pesticides on the adult longevity, progeny production and prey consumption of Cryptolaemus montrouzieri Mulsant (Coccinellidae, Coleoptera). Indian Journal of Plant Protection, v.25, n.1, p.48-51, 1997.

MAPA: Ministério da Agricultura, Pecuária e Abastecimento. Informe Estatístico do Café. 17p. 2007. Disponível em: <http://www.agricultura.gov.br>. Acesso em: 8 fev. 2008.

MIRET, J.A.J.; GARCÍA-MARÍ, F. Side effects of pesticides on selected natural enemies occurring in citrus in Spain. IOBCMPRS Bulletin, v.24, n.4, p.103112, 2001.

MORSE, J.G.; BELLOWS, T.S.J. Toxicity of major citrus pesticides to Aphytis melinus (Hymenoptera: Aphelinidae) and Cryptolaemus montrouzieri (Coleoptera: Coccinellidae). Journal of Economic Entomology, v.79. p.311-314, 1986.

MORSE, J.G.; BELLOWS, J.R.; GASTON, L.K.; IWATA, Y. Residual toxicity of acaricides to three beneficial species on California citrus. Journal of Economic Entomology, v.80, p.953-960, 1987. 
PETERSEN, H.; GJELSTRUP, P. Effects of dimethoate on Collembola growth and reproduction. Pesticides Research, n. $48,1998$.

\section{PMRA: PEST MANAGEMENT REGULATORY}

AGENCY. Nota regulatória: Thiamethoxam Helix, Helix XTra. Reg 2001-03. Health Canadá. Ottawa, 55p. 2001.

PUTTARUDRIAH, M.; CHANNABASAVANNA, G.P.; MURTI, B.K. Discovery of Cryptolaemus montrouzieri Mulsant (Coccinellidae, Coleoptera, Insecta) in Bangalore, South Índia. Nature, v.169, p.377-378, 1952.

RAIJ, B.V. Produção integrada de café - PIC. AgronômiCo, v.55, n.2, 2003.

RAMESH, T.; AZAM, U.M. Residual toxicity of different insecticides to the adult Cryptolaemus montrouzieri Mulsant (Coccinellidae: Coleoptera). Tropical Pest Management, v.33, n.2, p.180, 1987.

RIPPER, W.E. et al. Selective insecticides and biological control. Journal of Economic Entomology, v.44, n.4, p.448458, 1951.

SANTA-CECÍLIA, L.V.C; SOUZA, B.; SOUZA, J.C.; PRADO, E.; MOINO-JUNIOR, A.; FORNAZIER, M.J.; CARVALHO, G.A. Cochonilhas-farinhentas em cafeeiros: bioecologia, danos e métodos de controle. Belo Horizonte: Epamig. 2007. 48p. (Boletim Técnico, 79).

SAS INSTITUTE. SAS/STAT: users guide. Cary, NC. 2001.
SCOTT, A.J.; KNOTT, M.A. A cluster analyses method for grouping means in the analyses of variance. Biometrics, v.30, p.502-512, 1974.

SMITH, H.S.; ARMITAGE, H.M. Biological control of mealybugs in California. California Department of Agriculture Bulletin, v.9, p.104-158, 1920.

SMITH, S.F.; KRISCHIK, V.A. Effects of biorational pesticides on four coccinellid species (Coleoptera: Coccinellidae) having potential as biological control agents in interiorscapes. Journal of Economic Entomology, v.93, n.3, p.732-736, 2000.

VEIRE, van de; STERK, G.; STAAIJ, M. de van; RAMAKERS, P.M.J.; TIRRY, L. Sequential testing scheme for the assessment of the side-effects of plant protection products on the predatory bug Orius laevigatus. BioControl, v.47, n.1, p.101-113, 2002.

VOGT, H. Untersuchungen zu nebenwirkungen von insektiziden und akariziden auf Chrysoperla carnea Steph. (Neuroptera, Chrysopidae). Mededelingen Faculteit Landbouwkundige, v.57, n.2b, p.559-567. 1992.

WARE, G.W.; WHITACRE, D.M. An introduction to insecticides. 4.ed. Tucson, Arizona, 2004. Disponível em: <http://ipmworld.umn.edu/chapters/ware.htm>. Acesso em: 10 jul. 2007.

Recebido em 12/3/09

Aceito em 9/10/09 